\title{
Effects of massage on the expression of proangiogenic markers in rat skin
}

\author{
Katarzyna Ratajczak-Wielgomas, ${ }^{1}$, Krzysztof Kassolik ${ }^{2,3}$, Jedrzej Grzegrzolka', \\ Tomasz Halski ${ }^{3}$, Aleksandra Piotrowska ${ }^{1}$, Katarzyna Mieszala ${ }^{1}$, Iwona Wilk ${ }^{2}$, \\ Marzenna Podhorska-Okolow ${ }^{1}$, Piotr Dziegiel ${ }^{1,2}$, Waldemar Andrzejewski ${ }^{2,3}$
}

\author{
${ }^{1}$ Division of Histology and Embryology, Department of Human Morphology and Embryology, \\ Wroclaw Medical University, Wroclaw, Poland \\ ${ }^{2}$ Department of Physiotherapy, University School of Physical Education, Wroclaw, Poland \\ ${ }^{3}$ Public Higher Medical Professional School, Opole, Poland
}

\begin{abstract}
Introduction. Massage is a physiotherapeutic treatment, commonly used in both therapy and restoration of normal body functions. The aim of this work was to determine the effects of skin massage on stimulating the expression of angiogenesis-initiating factors, i.e. VEGF-A, FGF-2 (bFGF) and CD34 and on skin regeneration processes. Material and methods. The study was conducted on 48 Buffalo strain rats, randomly divided into two groups. In the first group (M, the massaged group), massage was applied five times a week for 7 weeks. In the second study group (C, the control group), the massage was omitted. Massage consisted of spiral movements at the plantar surface of skin for $5 \mathrm{~min}$ on each rear extremity. The gene expression of proangiogenic factors, including $V E G F-A, F G F-2, C D 34$ at the mRNA level was determined using real-time PCR. Immunohistochemistry was performed on paraffin sections of rat skin to determine VEGF-A, FGF-2 CD34 and Ki-67 expression.

Results. An increase in mRNA expression in the skin of the rat's rear extremity for VEGF-A and $F G F-2$ in the first week of the experiment was shown in the M group compared with the control rats. The upregulation of $C D 34$ mRNA expression was also observed in the $\mathrm{M}$ group. We observed positive correlations between $V E G F-A$ mRNA expression and the expression of mRNA for $F G F-2$ and $C D 34$, as well as correlation between the expression of mRNA for $F G F-2$ and $C D 34$. The immunohistochemical expression of VEGF-A, FGF-2 and CD34 was at a much lower level in the skin of control rats relative to the skin of massaged animals. Moreover, significantly higher immunoreactivity was shown for nuclear protein Ki-67 in epidermal cells in the M group compared with the $\mathrm{C}$ group.

Conclusions. Rat skin massage increased the expression of the main angiogenesis-stimulating factors and the proliferative activity of epidermal cells, which can stimulate skin regeneration and tissue repairing processes. (Folia Histochemica et Cytobiologica 2018, Vol. 56, No. 2, 83-91)
\end{abstract}

Key words: rat; skin; massage; VEGF-A; FGF-2; CD34; Ki-67; qPCR; IHC

\section{Introduction}

Massage has been used for a long time, as the integral part of physiotherapy, in prophylaxis, treatment, as well as in recovery of normal function of human body

Correspondence address: K. Ratajczak-Wielgomas Division of Histology and Embryology,

Department of Human Morphology and Embryology

Wroclaw Medical University,

Chalubinskiego 6a, 50-368 Wroclaw, Poland

tel.: +48 717841365 , fax: +48 717840082

e-mail: katarzyna.ratajczak-wielgomas@umed.wroc.pl after its impairment. Effects of massage are based on the commonly accepted outcomes, such as, inter alia, an increase in blood flow in the skin and muscles, normalisation of the autonomous system functions, increased secretion of some hormones, e.g. endorphins or cortisol, and lowering of blood pressure. It is also commonly believed, that systematically applied skin massage increases skin regeneration ability by improving its elasticity and resilience [1-4].

So far, there are no reports on the possible effects of massage as a skin angiogenesis-initiating factor, i.e. on the formation of new capillaries from the existing 
vascular network. Angiogenesis is a complex process that requires coordinated activity of a variety of vascular components: the division of endothelial cells, degradation of vascular basement membranes and the surrounding extracellular matrix, and endothelial cell migration. It is a multi-stage process regulated by many factors and it is the key element in wound healing and tissue regeneration $[5,6]$. The most important angiogenesis-stimulating factors include the family of Vascular Endothelial Growth Factors (VEGFs), with VEGF-A being the strongest stimulator. The effect of VEGF on the target cells is possible because of the presence of its specific receptors (VEGFRs): VEGFR-1 (Flt-1, Fms-like tyrosine kinase 1) and VEGFR-2 (KDR, Kinase Domain Region), located, inter alia, on the vascular endothelial cells [6,7]. The mechanisms of VEGF action in angiogenesis are multiple and can be seen on many levels. VEGF is an important chemoattractant, both for progenitor and mature endothelial cells. It induces proliferation, growth, and migration of endothelial cells and their spatial organisation during blood vessel formation. It also stimulates the expression of proteases engaged in extracellular matrix degradation [5, 6, 8]. Moreover, VEFG activates the expression of anti-apoptotic factors, and can, therefore, act as endothelial cells survival factor [5, 6]. Angiogenesis is also strongly stimulated by other growth factors, such as Basic Fibroblast Growth Factor (also known as bFGF or FGF-2), which is a ligand for the family of tyrosine kinase receptors, similarly to VEGF [8, 9]. FGF-2 increases the expression of angiopoietin-2, a potent regulator of vascular branching and angiogenesis, as well as VEGF in cultured human and endothelial cells [9]. FGFs can exert their angiogenic mechanism of action via paracrine signalling through their release by tumour and stromal cells or through their mobilization from the extracellular matrix (ECM). FGFs may also exert their effects through autocrine signalling in endothelial cells and stimulating the proliferation of new capillary endothelial cells. This induces a proangiogenic state that creates an environment favourable for vascular growth [9].

It is also worth noting, that angiogenesis intensity in a tissue can be directly assessed by the evaluation of microvessel density (MVD). In turn, transmembrane glycoprotein CD34 - sialomucin - is one of the markers of progenitor endothelial cells, which regulates migration of cells during formation of new vessels. CD34 appears on the cell surface at the early stages of endothelial cells formation and is present during their differentiation $[10,11]$. CD34 molecule is expressed on hematopoietic precursor cells, endothelial cells of small blood vessels, and cancer vessels. Therefore, its expression is associated with phenotype of primary vessels [12-14].

Our earlier publications showed that massage of tendons and skeletal muscles in rats, applied during long-term, repeated, intensive physical activity, has stimulatory effect on angiogenesis within the massaged tissues [15, 16]. Those studies show reliable arguments for the application of massage in order to improve the function of tendons and skeletal muscles, both in prophylactic and therapeutic treatment, as well as an element of biological recovery in sportsmen. Since tendon or skeletal muscle massage always involves skin over the massaged body region we decided to investigate how rat skin massage will affect the expression of angiogenesis-initiating factors, i.e. VEGF-A, FGF-2 and CD34. The secondary goal was to determine the effect of skin massage on skin regeneration processes. This may be important especially for the determination of the objective effects of massage on the stimulation of regeneration processes occurring in the skin, particularly during ageing, as massage is commonly used as a mean of, among others, increasing skin blood circulation for its better nutrition. So far, however, there are no reliable scientific studies to confirm those opinions.

\section{Materials and methods}

Animals and experimental design. The experiment was conducted at the Animal Research section of the Department of Pathomorphology, Wroclaw Medical University, Wroclaw, Poland. The study was conducted on 48 Buffalo strain rats, all ten month-old, randomly divided into two groups (24 rats in each group), housed in identical conditions. The animals arrived 3 weeks before experiments to allow them to adapt to the new environment. They were housed six per cage with free access to food and water. The light schedule was a $12: 12 \mathrm{~h}$ light-dark cycle and the ambient temperature was $20 \pm 2^{\circ} \mathrm{C}$. Massage consisted of spiral movements at the plantar surface of $1 \mathrm{~cm}^{2}$ of skin for $5 \mathrm{~min}$ on each rear extremity [17] as shown in Figure 1. During massage, the rats were held across the scapula and neck region in a hanging position. Control animals were handled in the same way as the experimental animals. In the first group ( $\mathrm{M}$, the massaged group), massage was applied five times a week for 7 weeks. In the second study group ( $\mathrm{C}$, the control group), the massage was omitted. In order to standardize the massage procedure, an algometer device (Digital Algometer Pain Diagnostic Gage, Wagner Instruments, Greenwich, CT, USA) of $0.5 \mathrm{~cm}^{2}$ area with a constant compression power of $9.81 \mathrm{~N}(1 \mathrm{~kg})$ was used.

Anaesthesia and sample collection. The study was approved by the Local Ethical Committee No. 1 for Animal Experiments in Wroclaw (decision no. 5/2011). All rats were 


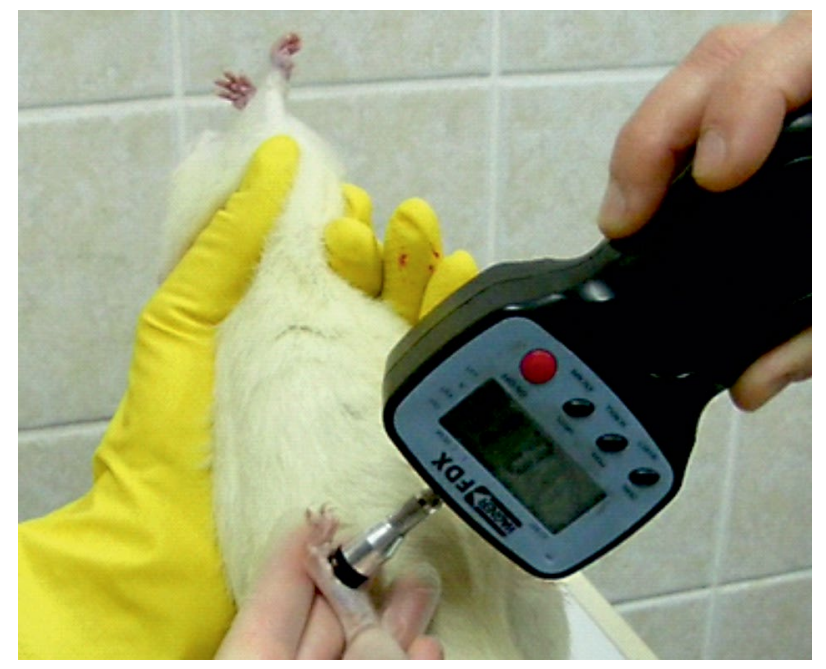

Figure 1. A photograph illustrates a massage of rat skin with an algometer device that was performed as described in Methods.

subjected to anaesthesia using ketamine $(10 \mathrm{mg} / \mathrm{kg}$ body weight) and sacrificed by decapitation. Tissue material for the study was sampled from 6 following rats from each experimental group at the end of weeks 1, 3, 5 and 7 of the experiment. Tissue samples collected from the skin of each rear extremity were fixed in $4 \%$ buffered formalin, dehydrated, and embedded in paraffin, as well as immediately immersed in liquid nitrogen and stored at $-80^{\circ} \mathrm{C}$. Paraffin sections were stained with haematoxylin and eosin (H\&E) and assessed by a pathologist under a BX41 light microscope (Olympus, Tokyo, Japan), whereas the frozen tissues were used for real-time PCR.

Immunohistochemistry. Immunohistochemical (IHC) reactions were performed using Autostainer Link 48 (Dako, Glostrup, Denmark). For the IHC reactions, paraffin blocks were cut into $4 \mu \mathrm{m}$-thick paraffin sections and fixed on sialinized microscopic slides. Deparaffinization and antigen retrieval were performed using Target Retrieval Solution (Dako) at $97^{\circ} \mathrm{C}$ for $20 \mathrm{~min}$ in a PT Link Rinse Station (Dako). Subsequently, skin sections were washed in TBS $/ 0.05 \%$ Tween and incubated with EnVision FLEX Peroxidase-Blocking Reagent (5 $\mathrm{min}$ at room temperature, RT). Then, the primary antibodies were applied for $20 \mathrm{~min}$ at RT. To detect VEGF-A expression, a mouse monoclonal antibody was used (1:200, Cat. No. M7273, Dako). The Ki67 antigen was detected using mouse monoclonal antibody, clone MIB-5 (ready-to-use; RTU, Dako); CD34 was detected with mouse monoclonal antibody (Cat. No. IR632, RTU, Dako) and FGF-2 with rabbit polyclonal antibody, (1:200, Cat. No. sc-79, Santa Cruz Biotechnology, Dallas, TX, USA). The sections were then washed in TBS $/ 0.05 \%$ Tween and
EnVision FLEX/HRP secondary antibodies (Dako) were applied for $20 \mathrm{~min}$ at RT. The peroxidase substrate, 3, 3'-diaminobenzidine (DAB), was applied and the sections were incubated for $10 \mathrm{~min}$ at RT. Finally, the sections were counterstained with Mayer's haematoxylin, and mounted in the SUB-X Mounting Medium (Dako). The expression of Ki-67 was assessed using Quant Centre 2.0 (3D Histech). In the epidermis, Ki-67-positive cells manifesting nuclear colour reaction were compared with all other epidermal cells.

RNA isolation and real-time PCR. Total RNA was isolated from the tissue samples using RNeasy Fibrous Mini Kit (Qiagen, Hilden, Germany) according to the manufacturer's protocol. To eliminate genomic DNA contamination, on-column DNase digestion was performed using RNaseFree DNase Set (Qiagen). The quantity and purity of RNA samples were assessed by measuring the absorbance at 260 and $280 \mathrm{~nm}$ with NanoDrop1000 spectrophotometer (Thermo Fisher Scientific, Wilmington, DE, USA). Reverse transcription reactions were performed with the use of High-Capacity cDNA Reverse Transcription kits (Applied Biosystems, Foster City, CA, USA). The expression of VEGF-A, CD34 and FGF-2 mRNA was determined by real-time PCR with the 7900 Fast-Real Time PCR System and iTag Universal Probes Supermix (Bio-Rad, Hercules, CA, USA) according to the manufacturer's protocol. $G A P D H$ was used as reference gene. For the reactions, the following sets of primers and TaqMan probes were used: Rn01511601_m1 for VEGF-A, Rn00570809_m1 for FGF-2, Rn03416140_m1 for CD34 and Rn99999916_s1 for GAPDH (Applied Biosystems). All reactions were performed in triplicate under the following conditions: initial denaturation at $94 \mathrm{C}$ for $10 \mathrm{~min}$ and 40 cycles of denaturation at $94^{\circ} \mathrm{C}$ for 15 sec, followed by annealing and elongation at $60^{\circ} \mathrm{C}$ for 160 sec. The relative expression of $V E G F-A, C D 34$ and $F G F-2$ mRNA (RQ) was calculated with the $\Delta \Delta \mathrm{Ct}$ method.

Statistical analysis. Statistical analyses were carried out using Prism 5.0 (GraphPad, La Jolla, CA, USA). The differences between the groups were tested using one-way ANOVA analysis of variance with Tukey post hoc test. The Kolmogorov-Smirnov test was applied to each group of data to test for normality. The Spearman correlation test was conducted to analyse the existing relationships. Results were considered statistically significant at $\mathrm{p}<0.05$.

\section{Results}

Expression of angiogenesis-initiating factors, including VEGF-A, FGF-2, CD34 at the mRNA level

In the first week of the experiment in the massaged group of rats, an increase ( $\mathrm{p}<0.01$, Fig. 2A) in $V E G F-A$ mRNA expression was found in comparison with the control group, in which constant level of ex- 


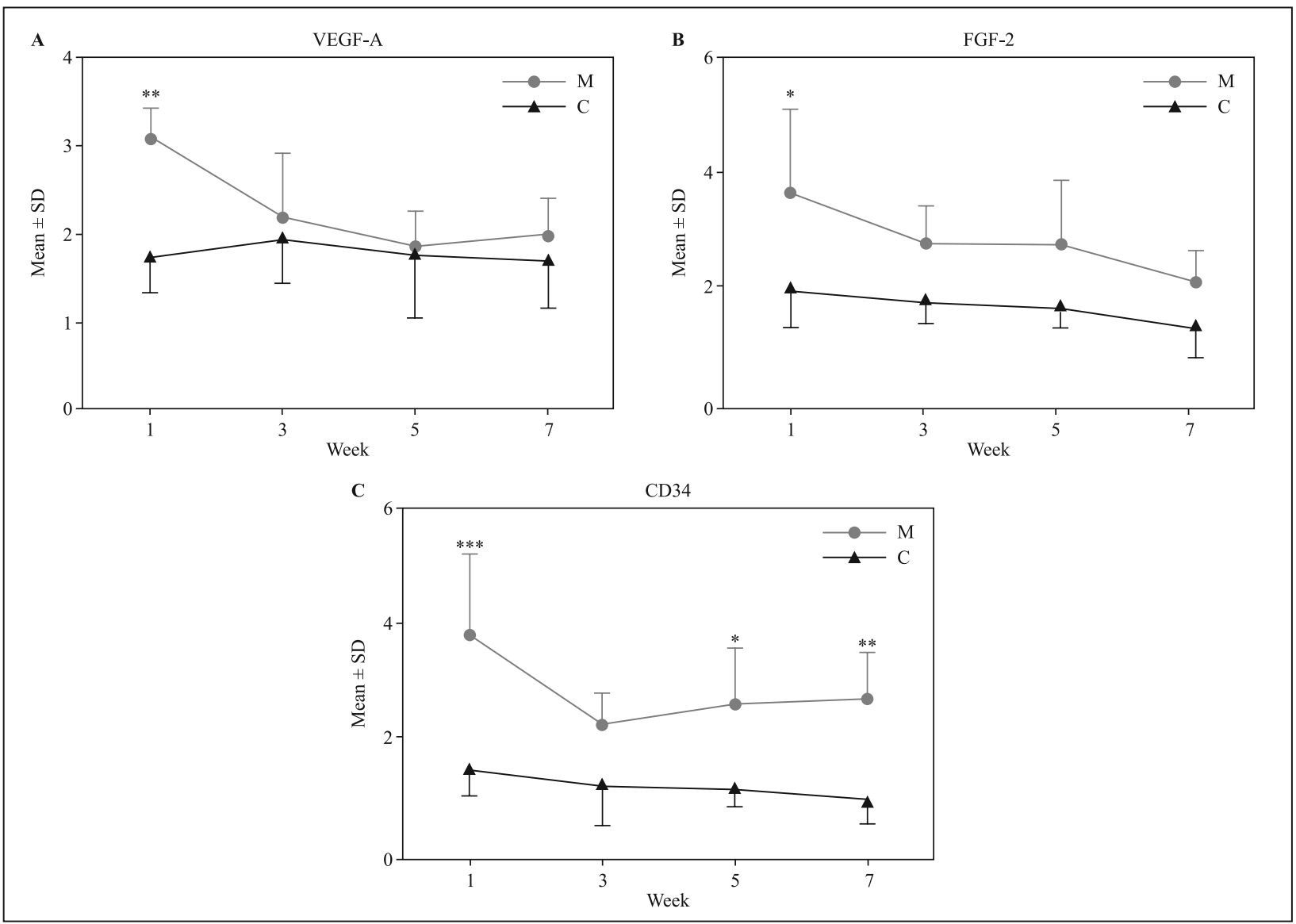

Figure 2. Differentiated mRNA expression of $V E G F-A(\mathbf{A}), F G F-2(\mathbf{B})$ and $C D 34(\mathbf{C})$ in the skin of massaged (M) and control (C) rats. Significant differences were noted between the $\mathrm{M}$ and $\mathrm{C}$ groups. ${ }^{*} \mathrm{p}<0.05,{ }^{* *} \mathrm{p}<0.01,{ }^{* * *} \mathrm{p}<0.001$; Tukey's post-hoc test.

pression was observed during the whole experiment. Slightly upward trend for VEGF-A remained also in the subsequent weeks of the experiment, and then, in the fifth week of the experiment, expression of mRNA for $V E G F-A$ was at the level similar to that of the control group (Fig. 2A). Similarly, the expression of $F G F-2$ at the mRNA level was higher $(\mathrm{p}<0.05$, Fig. $2 \mathrm{~B})$ in the first week of the experiment in the massaged group than in the control group. An increase in $F G F-2$ mRNA expression remained at the same level in the subsequent weeks of the experiment, i.e. in weeks 3 , 5 and 7. As shown in Figure 2C, increased expression of $C D 34$ mRNA in the massaged group was observed in week 1,5 and 7 relative to non-massaged animals ( $\mathrm{p}<0.001, \mathrm{p}<0.05, \mathrm{p}<0.01$, respectively).

The statistical analysis revealed positive correlation between the expression of mRNA for $V E G F-A$, and for $F G F-2$ and $C D 34(\mathrm{r}=0.5315$, $\mathrm{p}<0.0001$, $r=0.4390, p<0.0001$, Fig. 3A, B). A positive correlation was also noticed between the expression of $F G F-2$ mRNA and the expression of CD34 mRNA $(\mathrm{r}=0.5875, \mathrm{p}<0.0001$, Fig. 3C).

\section{Immunoreactivity of VEGF-A, FGF-2 \\ and CD34 in the massaged skin of rats}

The application of the IHC technique revealed in the skin of rats subjected to massage the presence of VEGF-A mainly in the cytoplasm of epidermal cells. Similar cellular localization was noticed for FGF-2. The immunoreactivity of CD34 was shown mainly in vascular endothelial cells (Fig. 4A, B, C). Due to the small size of skin samples available for the IHC analysis, no quantification of the studied angiogenic factors was performed. The immunoreactivity of VEGF-A, FGF-2 and CD34 was at a much lower level in the skin of control rats relative to the skin of massaged rats. Their expression in the skin of control rats is presented in Figures 4E, F, G.

\section{Evaluation of Ki-67 antigen expression}

The expression of the Ki- 67 proliferation antigen was found in the nuclei of epidermal cells, however, not in the skin proper (Fig. 4D). The expression of Ki-67 in the control group is shown in Figure 4H. Positive IHC reaction showing nuclear expression of $\mathrm{Ki}-67$ 


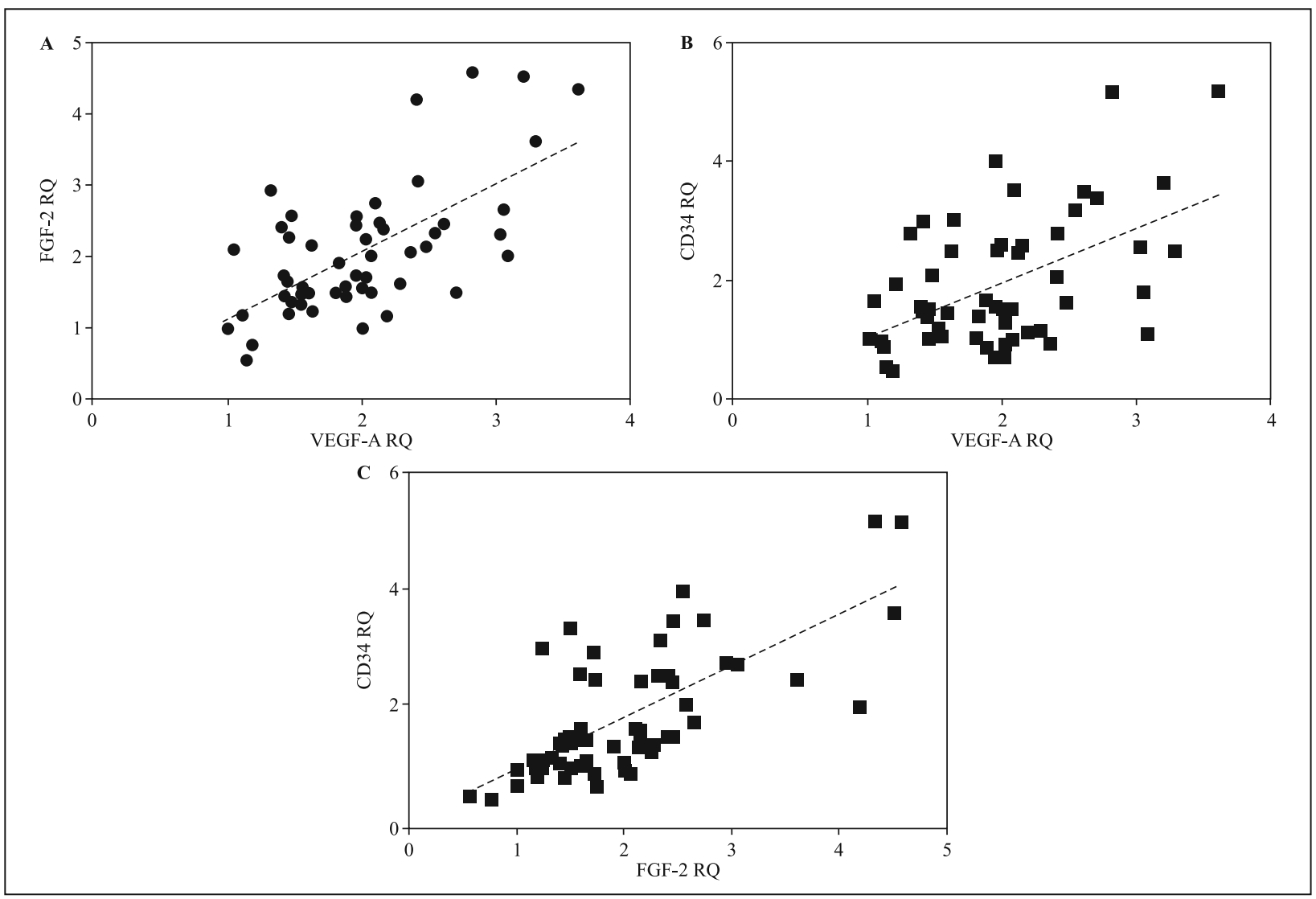

Figure 3. Correlations between the expressions of the studied genes at the mRNA level. A. $V E G F-A$ and $F G F-2$ gene expression in the group of massaged rats, $\mathrm{r}=0.5620, \mathrm{p}<0.0001$. B. $V E G F-A$ and $C D 34$ gene expression, $\mathrm{r}=0.4239$, $\mathrm{p}=0.0013$. C. $F G F-2$ and $C D 34$ gene expression, $\mathrm{r}=0.6916, \mathrm{p}<0.0001)$. Spearman correlation test. qPCR was performed as described in Methods.

antigen was found in 83 cases $(95.4 \%)$. Statistically significant higher level of Ki-67 expression in week 3 and 5 of the experiment was noticed in the epidermis of massaged rats as compared with the control animals $(\mathrm{p}<0.001, \mathrm{p}<0.05$, respectively; Fig. 5).

\section{Discussion}

So far, we are not aware of any studies showing beneficial effects of the skin massage on the angiogenesis process within the massaged tissue. Therefore, it is important to study whether long-term skin massage provides improvement to the skin vascularisation and nutrition, which may contribute to the increase in skin regeneration and potential adaptation mechanisms. It is believed, that VEGF-A and FGF-2 are one of most important regulatory factors in the angiogenesis process $[5,6,9]$.

Our studies showed higher expression of $V E G F-A$ mRNA in the skin samples of the massaged rats in comparison with the control, i.e. to the non-massaged animals. Moreover, we found increased expression of $F G F-2$ mRNA for and $C D 34$ mRNA in the massage group relative to the control rats in which expression remained constant during whole experiment. The statistical analysis showed positive correlations between $V E G F-A$ mRNA expression, and the expression of mRNA for FGF-2 and CD34, as well as significant positive correlation between the expression of mRNA for $F G F-2$ and $C D 34$. Comparable results were obtained by Erba et al. [18], who evaluated the effects of Vacuum Assisted Closure (VAC), as a form of mechanical skin deformation, on angiogenesis process. In the in vivo studies, in full-thickness wound obtained in dorsal region in mice, the increased density of CD31-marked blood vessels was shown [18]. It is in accordance with the trend observed in our studies, showing increased expression of the gene encoding CD34 in the massaged group of rats in comparison with the group which was not subjected to mechanical skin deformation (non-massaged group). Using Western blot technique, Erba et al. [18] also showed 


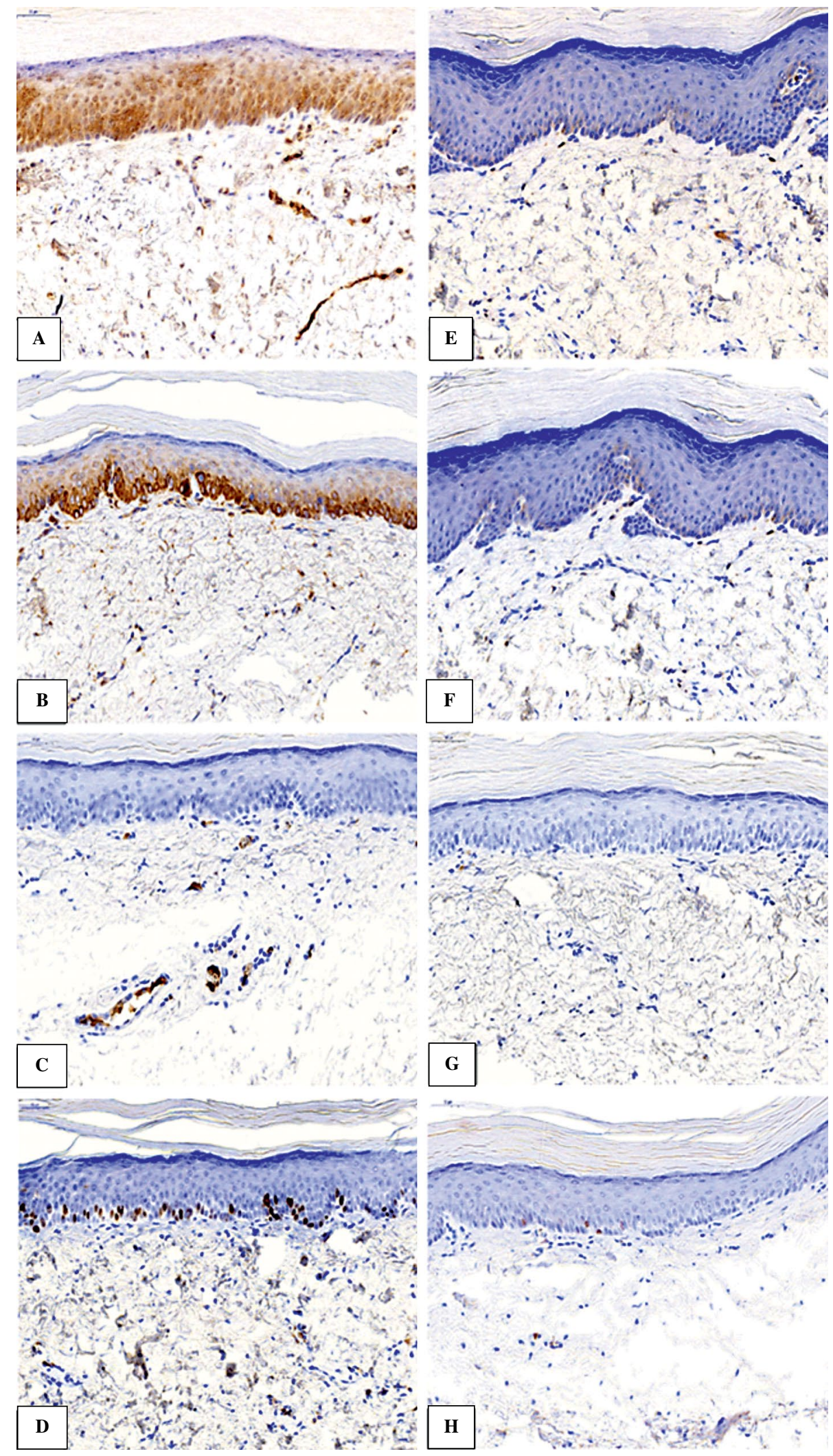

Figure 4. Immunohistochemical expression of VEGF-A (A), FGF-2 (B), CD34 (C) and Ki-67 (D) in the skin of massaged (A, $\mathbf{B}, \mathbf{C}, \mathbf{D})$ and control $(\mathbf{E}, \mathbf{F}, \mathbf{G}, \mathbf{H})$ rats. Skin samples were processed and stained by immunochemistry as described in Methods. 


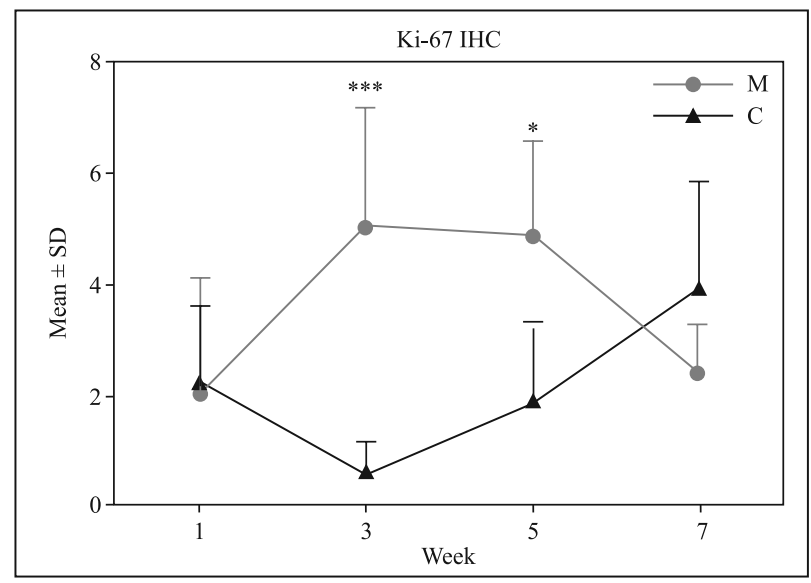

Figure 5. Semiquantitative evaluation of Ki-67 expression in the skin of massaged $(\mathrm{M})$ and control $(\mathrm{C})$ rats. ${ }^{*} \mathrm{p}<0.05$, $* * * \mathrm{p}<0.001$; Tukey's post-hoc test.

elevated expression of dimeric form of VEGF-A in the VAC treated wounds relative to the occlusive dressing control wounds. However, during the evaluation of expression of proangiogenic factors (VEGF-A, HIF- $1 \alpha$, PDGF-BB, VEGF-R2) at the mRNA level, results slightly different than ours were obtained. In the analysed group of mouse, mRNA expression of VEGF-A, HIF-1 $\alpha$, PDGF-BB, VEGF-R2 showed a progressive increase over the observation time, whereby control wounds were associated with a 15 fold increased expression of HIF- $1 \alpha$ and 5 fold increased expression of VEGF-A. The authors suggested, that it may be associated with the fact, that occlusive dressing control wounds showed generalized hypoxia, with associated accumulation of hypoxia-induced factor (HIF-1 $\alpha$ ) [18]. In turn, Xia et al. [19] analysed the effects of negative pressure wound therapy (NPWT), as a form of massage leading to the change in skin tension, on angiogenesis and regeneration in patients with a full-thickness infected wound. So far, there are only reports suggesting significant importance of NPWT in supporting the treatment of infected wounds; however, there are no data on the effects of NPWT on the process of proangiogenic factors stimulation.

Comparable to the results of our previous studies regarding the effect of massage on tendinous and muscle tissue in rats [15, 16], Xia et al. [19] showed increased expression of VEGF-A, EGF and PDGF at the protein level in the group of patients subjected to NPWT in the third day following the use of this therapy, relative to the patients who were not subjected to NPWT. The authors suggested that NPWT, as one of the forms of physiotherapy, may affect the local expression of angiogenesis-associated growth factors, which is also in line with the tendency observed in our studies. On the other hand, Jacob et al. [20], in a rodent full-thickness wound model, also showed elevated expression of two key angiogenesis stimulators (VEGF and FGF-2) at the protein level, following VAC therapy during first 5 days of the experiment. Additionally, using WB technique, increased expression of CD31 - a marker characteristic for blood vessels, was shown in the group of rats subjected to VAC therapy when compared with control wound covered only with an occlusive dressing. The obtained results clearly suggest the important effect of VAC therapy, as one of the forms of massage, on regeneration and angiogenesis processes, by, among others, increasing concentration of proangiogenic factors [20].

Also noteworthy are studies on the impact of using extracorporeal shock-wave therapy (ESTW) on wound and lesions [21, 22]. Stojadinovic et al. [23] investigated the role of extracorporeal shock-wave therapy (ESWT) in early revascularization of full-thickness skin isografts in a murine model. Authors suggest that early pro-angiogenic and anti-inflammatory effects of ESWT promote tissue revascularization and wound healing by augmenting angiogenesis [23]. In another study, Kuo et al. [24] showed that treatment with an ESWT significantly enhanced diabetic wound healing in a rat model associated with increased neo-angiogenesis and tissue regeneration. Kuo et al. [24] in immunochistochemical studies showed increased expression of angiogenic parameters, including VEGF and proliferating cell nuclear antigen (PCNA) in the ESWT group, as compared with the control animals. These results showed that ESTW, as a form of physiotherapy, may induce angiogenesis, by stimulating the expression of proangiogenic markers, which is consistently in line with the conclusions drawn from our studies. Comparable results were obtained by Asadi et al. [25], who made an attempt to evaluate expression of VEGF in in vivo studies in full-thickness wound model obtained by dorsal skin incision in rats subjected to electrical stimulation (ES). The authors showed higher expression of VEGF in the skin on the protein level (ELISA kit) in the seventh day of the experiment in the group of rats subjected to sensory ES $(600 \mu \mathrm{A})$ in comparison with the control group. This may suggest beneficial effects of ES, as one of the forms of physiotherapy, on angiogenesis, promoting wound healing and skin regeneration processes [25].

The obtained results are in line with our previous studies on the effect of massage of tendinous and skeletal muscle tissue on the angiogenesis process within massaged tissues $[15,16]$. Those studies showed significant increase in the expression of mRNA for $V E G F-A, C D 34$ and $F G F-2$ in the third week of the experiment in the group of rats subjected to tendon 
massage simultaneously with running training, which may suggest synergistic impact of simultaneous massage and physical exercise on the expression of angiogenesis markers in rat tendons [15]. Analogically, gradual increase in $V E G F-A$ mRNA expression was noticed also in case of skeletal muscle in the group massaged simultaneously with running training. This trend was maintained throughout the whole experiment and reached the statistical significance in relation to the control group of not massaged rats [16]. Those results allowed to assume that massage may contribute to processes of creation of new and development of already existing vascular networks in the skeletal muscle tissue and in rat tendons during increased exercise $[15,16]$. Similarly, in our study we showed that massage, as a form of mechanical stimulation, may induce the expression of angiogenic factors in the massaged skin of rats.

Significantly higher immunoreactivity was also shown for Ki-67 proliferative antigen in epidermal cells in the massaged group of rats in comparison with the control group. Ki-67 is a cell-cycle-associated antigen, expressed in all parts of the cell cycle except in G0 and early G1, and therefore confined to the proliferative compartment of the epidermis. $\mathrm{Ki}-67$ has proven to be of value as a marker of cell proliferation by recognizing the cell cycle-dependent expression of the Ki-67 non-histone nuclear antigen [26]. This suggests that skin massage may increase turnover rate of epidermal cells, which may be connected with an increase in regenerative capabilities and repair processes.

In summary, the obtained results may indicate beneficial effects of massage applied to the skin. The effect of mechanical stimulus in a form of massage on the skin increases expression of main angiogenesis-initiating factors within the massaged skin cells and induce their proliferation potential, which may indirectly create better conditions for regenerative processes.

\section{References}

1. Field T. Massage therapy research review. Complementary Therapies in Clinical Practice. 2016; 24: 19-31, doi: 10.1016/j. ctcp.2016.04.005.

2. Viravud Y, Apichartvorakit A, Mutirangura P, et al. The anatomical study of the major signal points of the courttype Thai traditional massage on legs and their effects on blood flow and skin temperature. J Integr Med. 2017; 15(2): 142-150, doi: 10.1016/S2095-4964(17)60323-6, indexed in Pubmed: 28285619.

3. Shin MS, Sung YH. Effects of Massage on Muscular Strength and Proprioception After Exercise-Induced Muscle Damage. J Strength Cond Res. 2015; 29(8): 2255-2260, doi: 10.1519/ /JSC.0000000000000688, indexed in Pubmed: 25226328.
4. Phuong C, Maibach H. Effect of massage on percutaneous penetration and skin decontamination: man and animal. Cutaneous and Ocular Toxicology. 2015: 1-4, doi: 10.3109/15569527.2015.1065501.

5. Hoff PM, Machado KK. Role of angiogenesis in the pathogenesis of cancer. Cancer Treat Rev. 2012; 38(7): 825-833, doi: 10.1016/j.ctrv.2012.04.006, indexed in Pubmed: 22677191.

6. De Palma M, Biziato D, Petrova TV. Microenvironmental regulation of tumour angiogenesis. Nat Rev Cancer. 2017; 17(8): 457-474, doi: 10.1038/nrc.2017.51, indexed in Pubmed: 28706266

7. Potente M, Gerhardt H, Carmeliet P. Basic and therapeutic aspects of angiogenesis. Cell. 2011; 146(6): 873-887, doi: 10.1016/j.cell.2011.08.039, indexed in Pubmed: 21925313.

8. Niu G, Chen X. Vascular endothelial growth factor as an anti-angiogenic target for cancer therapy. Curr Drug Targets. 2010; 11(8): 1000-1017, indexed in Pubmed: 20426765.

9. Lieu C, Heymach J, Overman M, et al. Beyond VEGF: inhibition of the fibroblast growth factor pathway and antiangiogenesis. Clin Cancer Res. 2011; 17(19): 6130-6139, doi: 10.1158/1078-0432.CCR-11-0659, indexed in Pubmed: 21953501.

10. Cao Y. VEGF-targeted cancer therapeutics-paradoxical effects in endocrine organs. Nat Rev Endocrinol. 2014; 10(9): 530-539, doi: 10.1038/nrendo.2014.114, indexed in Pubmed: 25048037.

11. Eichholz A, Merchant S, Gaya AM. Anti-angiogenesis therapies: their potential in cancer management. Onco Targets Ther. 2010; 3: 69-82, indexed in Pubmed: 20616958.

12. Ribatti D. The discovery of endothelial progenitor cells. An historical review. Leuk Res. 2007; 31(4): 439-444, doi: 10.1016/j.leukres.2006.10.014, indexed in Pubmed: 17113640.

13. Nagatsuka H, Hibi K, Gunduz M, et al. Various immunostaining patterns of CD31, CD34 and endoglin and their relationship with lymph node metastasis in oral squamous cell carcinomas. J Oral Pathol Med. 2005; 34(2): 70-76, doi: 10.1111/j.16000714.2004.00227.x, indexed in Pubmed: 15641985.

14. Corselli M, Chen CW, Crisan M, et al. Perivascular ancestors of adult multipotent stem cells. Arterioscler Thromb Vasc Biol. 2010; 30(6): 1104-1109, doi: 10.1161/ATVBAHA.109.191643, indexed in Pubmed: 20453168.

15. Andrzejewski W, Kassolik K, Dziegiel P, et al. Effects of synergistic massage and physical exercise on the expression of angiogenic markers in rat tendons. Biomed Res Int. 2014; 2014: 878095, doi: 10.1155/2014/878095, indexed in Pubmed: 24900996.

16. Andrzejewski W, Kassolik K, Kobierzycki C, et al. Increased skeletal muscle expression of VEGF induced by massage and exercise. Folia Histochem Cytobiol. 2015; 53(2): 145-151, doi: 10.5603/FHC.a2015.0013, indexed in Pubmed: 26150286.

17. Andrzejewski W, Kassolik K, Dziegiel P, et al. Massage may initiate tendon structural changes--a preliminary study. In Vivo. 2015; 29(3): 365-369, indexed in Pubmed: 25977382.

18. Erba P, Ogawa R, Ackermann M, et al. Angiogenesis in wounds treated by microdeformational wound therapy. Ann Surg. 2011; 253(2): 402-409, doi: 10.1097/ /SLA.0b013e31820563a8, indexed in Pubmed: 21217515.

19. Xia CY, Yu AX, Qi B, et al. Analysis of blood flow and local expression of angiogenesisassociated growth factors in infected wounds treated with negative pressure wound therapy. Mol Med Rep. 2014; 9(5): 1749-1754, doi: 10.3892/mmr.2014.1997, indexed in Pubmed: 24584462.

20. Jacobs S, Simhaee DA, Marsano A, et al. Efficacy and mechanisms of vacuum-assisted closure (VAC) therapy in promot- 
ing wound healing: a rodent model. J Plast Reconstr Aesthet Surg. 2009; 62(10): 1331-1338, doi: 10.1016/j.bjps.2008.03.024, indexed in Pubmed: 18617451.

21. Dymarek R, Halski T, Ptaszkowski K, et al. Extracorporeal shock wave therapy as an adjunct wound treatment: a systematic review of the literature. Ostomy Wound Manage. 2014; 60(7): 26-39, indexed in Pubmed: 25019247.

22. Zhang Li, Weng C, Zhao Z, et al. Extracorporeal shock wave therapy for chronic wounds: A systematic review and meta-analysis of randomized controlled trials. Wound Repair Regen. 2017; 25(4): 697-706, doi: 10.1111/wrr.12566, indexed in Pubmed: 28759136.

23. Stojadinovic A, Elster EA, Anam K, et al. Angiogenic response to extracorporeal shock wave treatment in murine skin isografts. Angiogenesis. 2008; 11(4): 369-380, doi: 10.1007/ /s10456-008-9120-6, indexed in Pubmed: 18998221.
24. Kuo YR, Wang CT, Wang FS, et al. Extracorporeal shockwave therapy enhanced wound healing via increasing topical blood perfusion and tissue regeneration in a rat model of STZ-induced diabetes. Wound Repair Regen. 2009; 17(4): 522-530, doi: 10.1111/j.1524-475X.2009.00504.x, indexed in Pubmed: 19614917.

25. Asadi MR, Torkaman G, Hedayati M. Effect of sensory and motor electrical stimulation in vascular endothelial growth factor expression of muscle and skin in full-thickness wound. J Rehabil Res Dev. 2011; 48(3): 195-201, indexed in Pubmed: 21480094.

26. Sezer E, Böer-Auer A, Cetin E, et al. Diagnostic utility of Ki-67 and Cyclin D1 immunostaining in differentiation of psoriasis vs. other psoriasiform dermatitis. Dermatol Pract Concept. 2015; 5(3): 7-13, doi: 10.5826/dpc.0503a02, indexed in Pubmed: 26336616.

Submitted: 8 November, 2017

Accepted after reviews: 8 May, 2018

Available as AoP: 14 May, 2018 